\title{
Interview mit dem neuen Direktor der WLB, Herrn Dr. Rupert Schaab
}

Am 1. Juni 2019 trat Dr. Rupert Schaab, der neue Leitende Bibliotheksdirektor der WLB, die Nachfolge des im vergangenen Jahr verstorbenen Dr. Hannsjörg Kowark an. Er war vorher in verschiedenen leitenden Funktionen an der Niedersächsischen Staats- und Universitätsbibliothek Göttingen tätig und freut sich auf die neue Herausforderung in Stuttgart. Im folgenden Gespräch äußert er sich zu seinen bisherigen Stationen, seinen Zielen und den wichtigsten aktuellen Aufgaben.

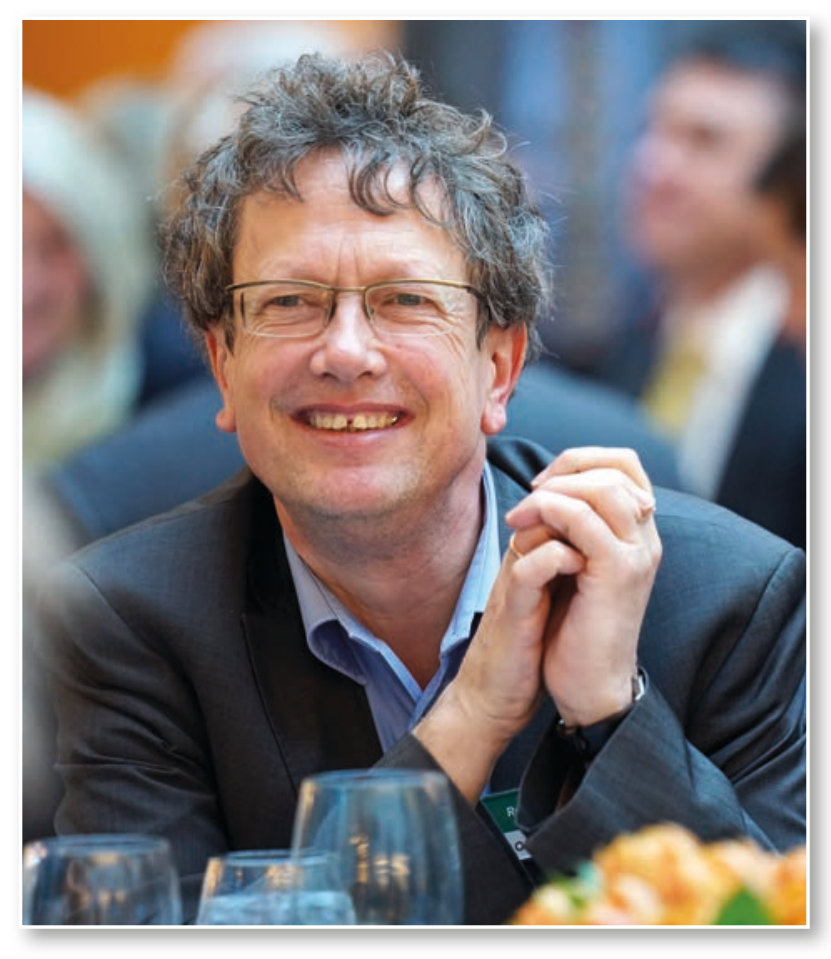

\section{Was hat Sie zu dem Beruf geführt?}

Wie viele Jugendliche war ich oft und gerne in Bibliotheken. Zunächst wollte ich nach meinem Zivildienst Medizin studieren und hatte nur aus Verlegenheit mit Germanistik und Geschichte in Heidelberg begonnen. In Bonn bekam ich dann eine Stelle bei einer Edition frühmittelalterlicher Bußbücher, schon damals datenbankbasiert, über ein Großrechnersystem! So wurde für mich der Umgang mit Handschriften und EDV schon früh wichtig. Vertieft wurde dies durch Studentenjobs in der Stiftsbibliothek St. Gallen. Hier entstand auch die Idee für meine Doktorarbeit zum Thema ,"Konvent und Schriftlichkeit im frühmittelalterli- chen Sankt Gallen (800 - 933)“. Mein Doktorvater empfahl mir dann aus familiären Gründen ein Bibliotheksreferendariat aufzunehmen, das mich 1993 nach Tübingen führte. Neben dem Rat meiner Oma, Andrea sei die Richtige, einer der besten Ratschläge, die ich bekommen habe.

\section{Wie ging es nach dem Referendariat weiter?}

Meine erste Stelle trat ich in Erfurt an, in einer Universitätsbibliothek im Aufbau. Die gestalterischen Möglichkeiten waren entsprechend vielfältig. Die Haltung "Das haben wir immer so gemacht" zählte nicht. Umfangreiche Buchbestände wurden in eine Systematik gebracht, ein Thema, das mich auch aktuell wieder begleitet. Besonders reizvoll war die Verantwortung, die ich für die Forschungsbibliothek Gotha erhielt. Im Zentrum standen große Projekte im Bereich der historischen Überlieferung. Es ging um die Erschließung von Handschriften und alten Drucken, die Realisierung von Jahresausstellungen und das Einwerben von Stipendienprogrammen.

\section{Welches Projekt fanden Sie in dieser Zeit besonders reizvoll?}

Hervorheben möchte ich die Erwerbung der kartografischen Sammlung des Justus Perthes Verlags mit mehr als 200.000 Blatt. Sie enthält einzigartiges Quellenmaterial zur Entwicklung der Kartografie und Geografie im 19. und 20. Jahrhundert. Die Sammlung wurde 2003 mit Unterstützung der Kulturstiftung der Länder erworben und in die Forschungsbibliothek Gotha integriert. Hinter der Reinigung und Erschließung der Bestände steht eine Vielzahl an effizient gestalteten Arbeitsprozessen. Die Arbeit an großen, über Dritt- und Sondermittel geförderten Projekten hat meine Tätigkeit in Erfurt und Gotha maßgeblich begleitet und mich neue Herausforderungen suchen lassen. Das führte mich nach Göttingen.

\section{Warum Göttingen?}

Göttingen besitzt eine attraktive Universität und die Bibliothek ist an vielen spannenden Projek- 
ten beteiligt. Neben administrativen Aufgaben traten insbesondere große Bauprojekte wie die 2006 abgeschlossene Sanierung des Historischen Gebäudes, die es ermöglichte, die Spezialsammlungen der Bibliothek an einem Ort sichtbar zu machen, aber auch die Neustrukturierung von zwei Fakultätsbibliotheken. Gestalterische Fragen sowie die Auseinandersetzung mit den Nutzungsaspekten von Neubauten gehörten zum Tagesgeschäft. Hinzu kam die Massendigitalisierung im Zusammenhang mit den großen nationalbibliographischen Projekten für das 17. und 18. Jahrhundert. Wichtig war ferner die Mitarbeit an der Deutschen Digitalen Bibliothek, ein spartenübergreifendes Portal, in das Archive, Bibliotheken und Museen ihre Objekte einspielten. Und schließlich lernte ich die internationale Zusammenarbeit schätzen, bei der ich neun Jahre lang deutsche Interessen bei der Bibliotheksautomatisierung und dem WorldCat wahrnehmen durfte. Gerade im internationalen Austausch habe ich viel gelernt.

\section{Gab es neben den vielen positiven Erfahrun- gen auch Entwicklungen, denen Sie kritischer gegenüberstehen?}

Nicht sehr glücklich war ich über die Reform der Sondersammelgebiete bzw. ihren Umbau in Fachinformationsdienste (FID), die den Fokus auf kurzfristige Informationsbeschaffung richtete. Gute unikale Bestände lassen sich aber nur langfristig aufbauen, benötigen Kontinuität und eine weiterreichende Sicherstellung der Mittel. Die Ausrichtung der alten Sondersammelgebiete erlaubte es, Literatur vorsorglich zu erwerben, was gerade für die geisteswissenschaftlichen Fächer und die kleinen, seltenen Disziplinen sehr wichtig ist. Die Grundidee der FIDs ließ sich damit nicht recht vereinbaren.

\section{Hätten Sie in dieser Zeit gedacht, wieder nach Baden-Württemberg bzw. nach Stuttgart zu kommen?}

Stuttgart ist in der Göttinger Zeit ganz aus dem Blick geraten. Als ich aber 2018 die Stellenausschreibung der WLB las, reizte mich die neue Aufgabe sofort, nicht zuletzt angesichts der besonderen unikalen Sammlungen der Bibliothek und dem großen Bauprojekt. Viele meiner Erfahrungen passen hierzu, und ich bin glücklich, dass ich die Möglichkeit erhalten habe.

\section{Was waren Ihre ersten Eindrücke?}

Auffallend war die große Herzlichkeit, mit der ich hier empfangen wurde, sowohl in der Bibliothek, aber auch seitens der anderen Kultureinrichtungen. Es entspricht nicht unbedingt dem Klischee, das man vom Schwaben hat. Der Besuch vieler Einrichtungen bildete einen wichtigen Schwerpunkt der ersten Monate. Vom vielfältigen Interesse an einer stärkeren Zusammenarbeit war ich angenehm überrascht. Und der Rückhalt der Bibliothek in der Württembergischen Bibliotheksgesellschaft sucht wohl - zumindest in Deutschland - seinesgleichen.

\section{Wie empfanden Sie bisher die Gespräche hier im Haus?}

Vieles in den Gesprächen begegnet einem auch andernorts. Erfreulich fand ich das überdurchschnittliche Engagement und die lebhafte Beteiligung der Bereiche. Und natürlich spüre ich auch die Unsicherheiten über die Absichten des neuen Direktors und die Sorge, nicht die erforderliche Aufmerksamkeit zu finden.

\section{Welche Schwerpunkte setzen Sie für die nächs- te Zeit?}

Natürlich steht das aktuelle Bauprojekt im Zentrum. Der Abschluss aller Arbeiten und der Bezug des Erweiterungsbaus sind zurzeit die größte Herausforderung. Die sich anschließende Sanierung des Bestandsgebäudes muss unter vielen anderen Bauvorhaben des Landes seinen Platz finden. Denn zahlreiche Missstände wie der nicht ausreichende Brandschutz, vielfache Undichtigkeiten im Magazin sowie die mangelhafte Klimatisierung müssen unbedingt behoben werden. Hinzu kommen die Vorbereitungen für den Umzug, um das Gebäude freizuziehen, gleichzeitig aber auch den Platz für gut 4 Millionen Bücher und zahlreiche Büros zu finden, ohne den Benutzungsbetrieb zu beeinträchtigen, und das im engen Stuttgart - wahrlich nicht einfach! Starke Unterstützung erhalten wir hier vom Amt für Vermögen und Bau.

\section{Welche Projekte stehen noch im Fokus?}

Der Erweiterungsbau bietet attraktive große Räumlichkeiten für Veranstaltungen und Ausstellungen. So prominent wie er an der Konrad-Adenauer-Straße steht, wäre er ein idealer Platz für den Austausch zwischen den Wissenschaften und 
der Öffentlichkeit. Stuttgart ist nicht nur während eines Festivals eine Wissenschaftsstadt. Nennen möchte ich aber auch die Digitalisierung, vor allem im Bereich der Zeitungen, wo die Papiere rapide verfallen. Und natürlich müssen auch die anderen digitalen Angebote in unserem Portfolio noch ausgebaut werden.

\section{Wie sehen Sie das Verhältnis von analog und digital?}

Hier sollte mit Blick auf die Leserbedürfnisse und den Inhalt genau unterschieden werden, wie ich selbst im Rahmen einer größeren wissenschaftlichen Arbeit erfahren durfte. Auch hier gilt: nicht jeder Inhalt ist für jedes Medium gleichermaßen geeignet. Die Bequemlichkeit und Nutzungsmöglichkeiten des Digitalen will niemand missen, eine intensive Auseinandersetzung mit vielen Textsorten ist aber oft besser auf Papier möglich.

\section{Herzlichen Dank für das Gespräch!}

Jörg Ennen / Rupert Schaab

\section{Kurzbiografie von Dr. Rupert Schaab}

1982 Abitur am Kurfürst-Friedrich-Gymnasium in Heidelberg,

1984-1990 Studium der Geschichte, Germanistik, Historischen Hilfswissenschaften und Philosophie in Heidelberg und Bonn,

1993-1995 Bibliotheksreferendariat an der Universitätsbibliothek Tübingen und der Fachhochschule Köln,

1996 Fachreferent an der Universitätsbibliothek Erfurt,

1998 Promotion an der Universität Bonn (Mönch in St. Gallen - Zur inneren Geschichte eines frühmittelalterlichen Klosters),

1999 Leitung der Forschungsbibliothek Gotha,

2005 stellvertretender Direktor an der Niedersächsischen Staats- und Universitätsbibliothek Göttingen.

Publikationen zur lateinischen Bibel im Mittelalter, zum frühmittelalterlichen Mönchtum, zur höfischen Kultur in der frühen Neuzeit sowie zur Überlieferungssicherung.

Langjährige Vertretung von Bibliotheksinteressen im Global Council von OCLC (WorldCat), der Deutschen Digitalen Bibliothek und der Allianz Schriftliches Kulturgut Erhalten! 